\title{
Antiferromagnetically Exchange-coupled Two Phase Magnets: $\mathrm{Co} / \mathrm{Co}_{2} \mathrm{TiSn}$
}

\author{
Taewan Kim ${ }^{1 *}$ and Jung Keun $\mathrm{Oh}^{2}$ \\ ${ }^{1}$ Department of Advanced Materials Engineering, Sejong University, Seoul 143-747, Korea \\ ${ }^{2}$ College of Architecture, Konkuk University, Seoul 143-701, Korea
}

(Received 4 March 2008)

\begin{abstract}
The objective of this paper is to review the magnetic and magneto-transport properties of $\mathrm{Co}_{\mathrm{C}} \mathrm{Co}_{2} \mathrm{TiSn}$ consisting of two metallic magnetic phases that are antiferromagnetically exchange-coupled at the phase boundary. The bulk $\mathrm{Co} / \mathrm{Co}_{2} \mathrm{TiSn}$ system, which has a $\mathrm{Co}_{2} \mathrm{TiSn}$ Heusler alloy precipitates in the hexagonal Co matrix, showed an unusual coercivity change with a concurrent change in temperature, and was modeled on the basis of a wall formation caused by exchange coupling at the phase boundary. For measurements of magneto-transport properties, $\mathrm{Co} / \mathrm{Co}_{2} \mathrm{TiSn}$ thin films that had two-magnet phases were deposited using a magnetron sputtering system with a composite target. The magnetization process in the films is also explained on the basis of the model of wall formation at the phase boundary. Annealed $\mathrm{Co} / \mathrm{Co}_{2} \mathrm{TiSn}$ films showed a $0.12 \%$ GMR effect, indicating the scattering of polarized conduction electrons due to the antiparallel exchange coupling at the phase boundary. The scattering process of conduction electrons at the phase boundary was modeled with relation to the magnetization process.
\end{abstract}

Keywords : two phase magnet, antiparallel exchange, wall formation giant magnetoresistance

\section{Introduction}

In two-phase magnets, where the two phases are in intimate contact with each other, it is possible for exchange coupling to occur at the phase boundary. The two-metallic phase magnets are materials that consist of two metallic magnetic phases with a negative or positive exchange at the phase boundary. For example, the exchange-spring magnet with a hard phase $\mathrm{Nd}_{2} \mathrm{Fe}_{14} \mathrm{~B}$ in soft $\mathrm{Fe}_{23} \mathrm{~B}_{6}+\mathrm{Fe}_{3} \mathrm{~B}$ has been proposed in order to create permanent magnets from composite materials that consist of two suitably dispersed ferromagnetic and mutually exchange-coupled phases. One of these phases is a hard magnet that will provide a high coercivity, while the other phase is a soft magnet providing a high magnetization [1]. Other similar system using a direct exchange-coupling at the interface between hard and soft magnetic layers has demonstrated the formation of domain wall, which was caused by the antiferromagnetic exchange-coupling at the interface between two magnetic layers [2]. In a $\mathrm{Co} / \mathrm{Co}_{2} \mathrm{MnSn}$ twophase magnet, one of the metallic phases is a low $T_{c}$ and low magnetization phase, hexagonal Co solid solution,

*Corresponding author: Tel: +82-2-3408-3691

Fax: +82-2-3408-3664, e-mail: twkim@ sejong.ac.kr while the other metallic phase is a high $\mathrm{T}_{\mathrm{c}}$ and high magnetization phase, $\mathrm{Co}_{2} \mathrm{MnSn}$ solution. The Co solid solution precipitates in the $\mathrm{Co}_{2} \mathrm{MnSn}$ Heusler alloy matrix [3]. In this system it has been shown that the Co solid solution precipitates are crystallographically coherent with the matrix and that exchange coupling occurs at the phase boundary. A macroscopic ferrimagnet Co- $\mathrm{TbN}$ film showed that the $\mathrm{TbN}$ particles are antiferromagnetically exchange coupled to the Co matrix, at least at the $\mathrm{Co} / \mathrm{TbN}$ interface $[4,5]$. The $\mathrm{Co}-\mathrm{TbN}$ system showed a giant magneto-resistance (GMR) effect, which is induced by the rotation of antiferromagnetically exchange-coupled spins at the phase boundary under the magnetic field.

A study was carried out on the $\mathrm{Co} / \mathrm{Co}_{2} \mathrm{TiSn}$ two-phase magnets in an effort to form a macroscopic ferrimagnet consisting of two metallic ferromagnetic phases, which are antiparallel exchange coupled at the phase boundary. The $\mathrm{Co} / \mathrm{Co}_{2} \mathrm{TiSn}$ system has a low $\mathrm{T}_{\mathrm{c}}, \mathrm{Co}_{2} \mathrm{TiSn}$, and a high $\mathrm{T}_{\mathrm{c}}$, hexagonal Co. The $\mathrm{Co}_{2} \mathrm{TiSn}$ Heusler alloy precipitates out of the hexagonal Co matrix. This system showed an unusual coercivity change with a concurrent change in temperature, and was modeled on the basis of the wall formation caused by exchange coupling at the phase boundary. The $\mathrm{Co} / \mathrm{Co}_{2} \mathrm{TiSn}$ thin films exhibited a giant magnetoresistance (GMR), providing evidence of anti- 
parallel exchange coupling occurring at the phase boundary. The model of the wall formation at the phase boundary was introduced in order to explain the GMR effect of $\mathrm{Co} / \mathrm{Co}_{2} \mathrm{TiSn}$ thin films.

\section{Experiments}

Ingots were prepared by arc melting in a commercial arc furnace with a water-cooled hearth and tungsten electrode. The chamber was evacuated by a mechanical pump and back filled several times with argon. The high purity argon gas (99.999\%) flowed through the chamber continuously during arc melting. An analysis of the microstructures was carried out with backscattered electron and secondary electron images on a secondary electron microscopy (SEM). A compositional analysis of the phases was carried out using energy dispersive $\mathrm{x}$-ray (EDX) analysis. The samples with two phases were characterized by $\mathrm{x}$-ray diffraction analysis. In the annealing experiments of ascast bulk samples, the samples were annealed for 48 hours at $870^{\circ} \mathrm{C}$ in an $\mathrm{N}_{2}$ gas atmosphere, which prevents the decomposition of the intermetallic compound by the oxidation. The $\mathrm{Co} / \mathrm{Co}_{2} \mathrm{TiSn}$ thin films were deposited with 5 15 mTorr Ar pressure using a magnetron sputtering system with a composite target that had a target composition of $\mathrm{Co}_{4} \mathrm{TiSn}$. The annealing was carried out for 4 hours in a vacuum and an $\mathrm{N}_{2}$ gas atmosphere. The magnetization loops and magnetization vs. temperature were made in the temperature range of $-100^{\circ} \mathrm{C}$ to $400^{\circ} \mathrm{C}$ up to a $13 \mathrm{kOe}$ field using a vibrating sample magnetometer (VSM). The magnetoresistance and Hall effect measurements taken at room temperature have been carried out in fields up to $8 \mathrm{kOe}$ with a DC electromagnet using Van der Pauw geometry.

\section{Results and Discussion}

3.1. The phase equilibriums and magnetic properties with Co composition along the pseudo-join $\mathrm{Co}-\mathrm{Co}_{2} \mathrm{TiSn}$

SEM pictures show that the $\mathrm{Co}_{2} \mathrm{TiSn}$ Heusler alloy becomes the primary phase at $60 \sim 78$ atomic $\%$ of $\mathrm{Co}$ and crystallizes out of a hexagonal Co matrix. At 60-71 atomic $\%$ of $\mathrm{Co}\left(\mathrm{Co}_{3} \mathrm{TiSn}, \mathrm{Co}_{4} \mathrm{TiSn}, \mathrm{Co}_{5} \mathrm{TiSn}\right)$, there is no significant change in the size of the $\mathrm{Co}_{2} \mathrm{TiSn}$ Heusler alloy precipitates and only the volume fraction of the precipitate is changed. At 78 atomic $\%$ of $\mathrm{Co}\left(\mathrm{Co}_{7} \mathrm{TiSn}\right)$, the size of the precipitates significantly decreases. The x-ray diffraction from the $\mathrm{Co}_{4}$ TiSn sample shows a stable two-phase $\mathrm{Co} / \mathrm{Co}_{2}$ TiSn (Fig. 1). In a comparison between the peaks from the arc-melted pure hexagonal $\mathrm{Co}$ and the one-phase $\mathrm{Co}_{2}$ TiSn Heusler alloy, the diffraction peaks from the

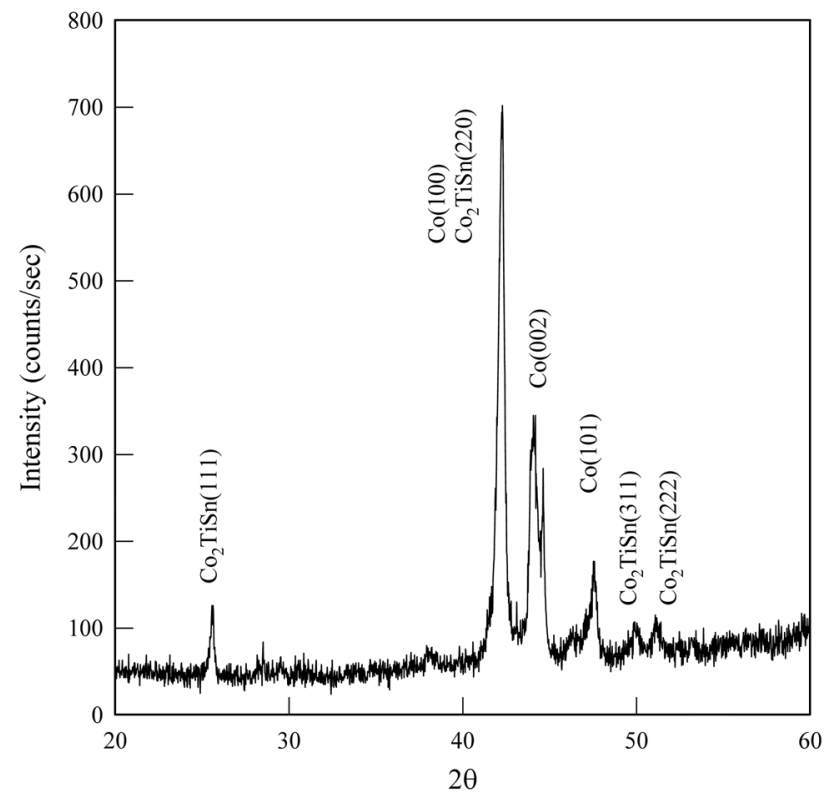

Fig. 1. The x-ray diffraction pattern from the $\mathrm{Co}_{4} \mathrm{TiSn}$ composition with two phases (hexagonal $\mathrm{Co}$ and $\mathrm{Co}_{2} \mathrm{TiSn}$ ).

$\mathrm{Co}_{4} \mathrm{TiSn}$ match those of the hexagonal $\mathrm{Co}$ and $\mathrm{Co}_{2} \mathrm{TiSn}$. The (100) peak of the hexagonal Co matrix overlaps with the (220) peak of the $\mathrm{Co}_{2} \mathrm{TiSn}$ Heusler alloy precipitates. There is no shift in the peak positions for the hexagonal Co matrix or for the $\mathrm{Co}_{2} \mathrm{TiSn}$ Heusler alloy precipitates.

The saturation magnetization, $\mathrm{M}_{\mathrm{s}}\left(\mathrm{emu} / \mathrm{cm}^{3}\right)$, vs. the volume fraction of Co to $\mathrm{Co}_{2} \mathrm{TiSn}$ is shown in Fig. 2. Line ' $\mathrm{a}$ ' is the calculated magnetization based on the volume fraction of $\mathrm{Co}$ to $\mathrm{Co}_{2} \mathrm{TiSn}$, while the magnetization line ' $b$ ' is obtained from experimental data. The magnetization is linearly proportional to the Co volume

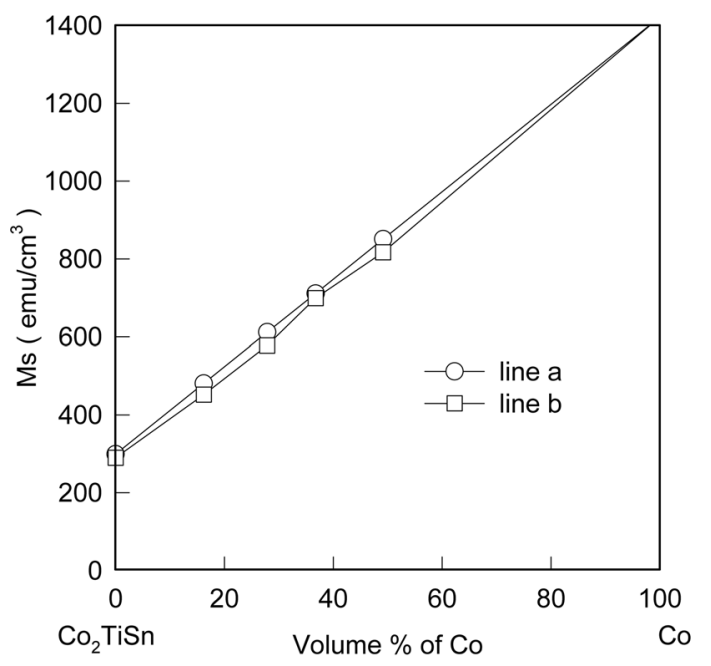

Fig. 2. Saturation magnetization, $\mathrm{Ms}\left(\mathrm{emu} / \mathrm{cm}^{3}\right)$, as a function of the volume fraction ratio of $\mathrm{Co}$ to $\mathrm{Co}_{2} \mathrm{TiSn}$ : a) theoretical magnetization, b) experimental magnetization. 


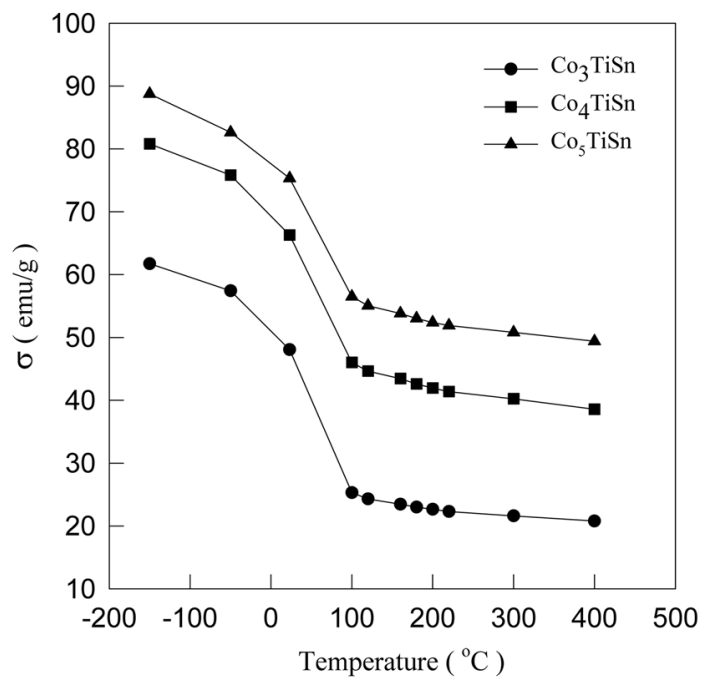

Fig. 3. Magnetization vs. temperature curves of $\mathrm{Co}_{3} \mathrm{TiSn}$, $\mathrm{Co}_{4} \mathrm{TiSn}$, and $\mathrm{Co}_{5} \mathrm{TiSn}$.

fraction.

\subsection{The unusual change of coercivity and initial mag-} netization curves with change in temperature

Fig. 3 and Fig. 4 show the magnetization and coercivity vs. temperature for three compositions, those of $\mathrm{Co}_{3} \mathrm{TiSn}$, $\mathrm{Co}_{4} \mathrm{TiSn}$, and $\mathrm{Co}_{5} \mathrm{TiSn}$, at which the volume fraction of Co increases with the Co content. It can be seen from Fig. 3 that the samples contain two magnetic phases: one with a low Curie temperature $\left(\mathrm{T}_{\mathrm{c}}\right)$ and the other with a high Curie temperature $\left(T_{c}\right)$. The $T_{c}$ with the low Curie temperature phase can be estimated by extrapolating from the break in the magnetization curves (Fig. 3). The break point for all the magnetization curves are at almost the same temperature, which is consistent with the Curie temperature of $\sim 100^{\circ} \mathrm{C}$, of the arc-melted one-phase $\mathrm{Co}_{2}$ TiSn Heusler alloy. It can be seen from Fig. 4 that, similarly to the case of magnetization vs. temperature, the coercivities in the $\mathrm{Co}_{3} \mathrm{TiSn}, \mathrm{Co}_{4} \mathrm{TiSn}$, and $\mathrm{Co}_{5} \mathrm{TiSn}$ samples, as a function of temperature, can be divided into low and high coercivity regions with approximately $100^{\circ} \mathrm{C}$ at the center. The Curie temperatures, determined from the temperature at which $\mathrm{H}_{\mathrm{c}}(\mathrm{T})$ changes, are approximately $100^{\circ} \mathrm{C}$ and approximately concur with the $\mathrm{T}_{\mathrm{c}}$ of the $\mathrm{Co}_{2} \mathrm{TiSn}$ phase obtained from the magnetization vs. temperature data. The coercivity decreases according to temperature in the low temperature region, and suddenly increases at the temperature in which the magnetization of the low $\mathrm{T}_{\mathrm{c}}$ $\mathrm{Co}_{2} \mathrm{TiSn}$ phase disappears. Arc-melted one-phase $\mathrm{Co}_{2} \mathrm{TiSn}$ Heusler alloy shows a closed loop without coercivity or remanence. The unusual temperature dependence of the coercivity can be explained by exchange coupling at the phase boundary.

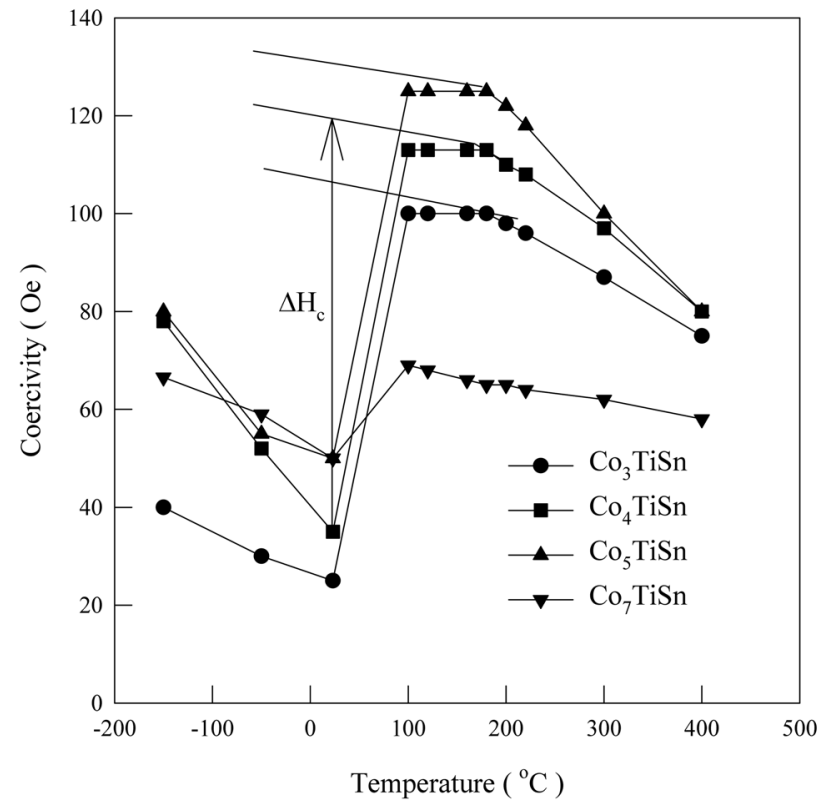

Fig. 4. Coercivity vs. temperature curves of $\mathrm{Co}_{3} \mathrm{TiSn}, \mathrm{Co}_{4} \mathrm{TiSn}$, $\mathrm{Co}_{5} \mathrm{TiSn}$, and $\mathrm{Co}_{7} \mathrm{TiSn}$.

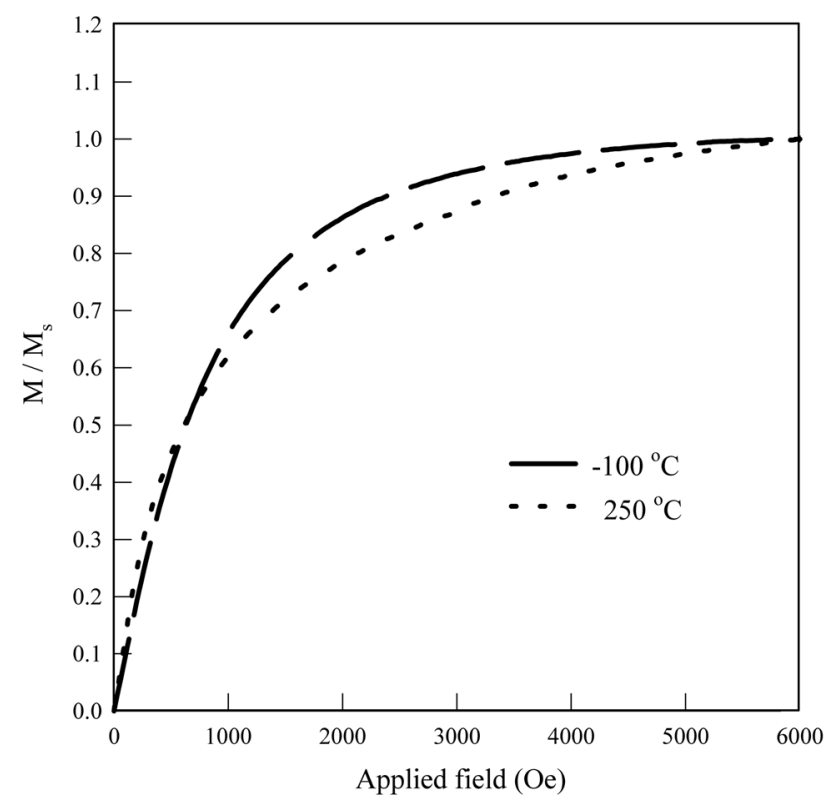

Fig. 5. Initial magnetization curves of $\mathrm{Co}_{4} \mathrm{TiSn}$ at high and low temperatures.

Fig. 5 shows the normalized initial magnetization curves of the $\mathrm{Co}_{4} \mathrm{TiSn}$ sample obtained at temperatures of -100 ${ }^{\circ} \mathrm{C}$ and $250^{\circ} \mathrm{C}$. It can be seen from the shape of the initial magnetization curves that the magnetization at a high temperature occurs by a different process from that occurring at a low temperature.

3.3. The model of unusual change in coercivity with temperature 
a) Below $T_{c}$

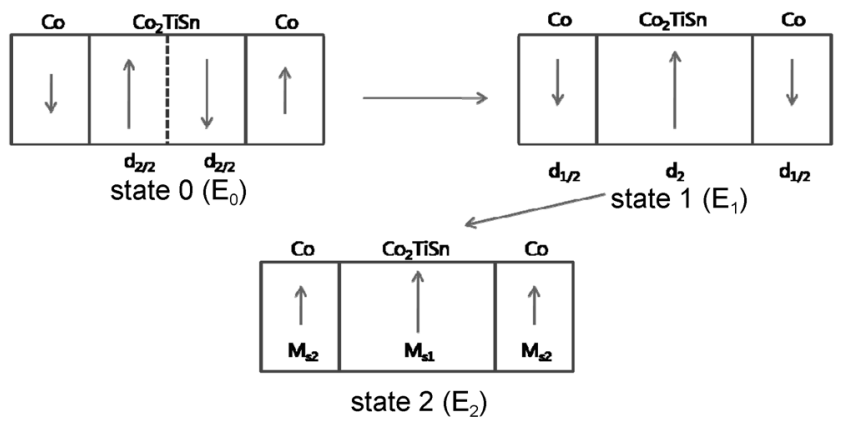

b) Above $T_{c}$
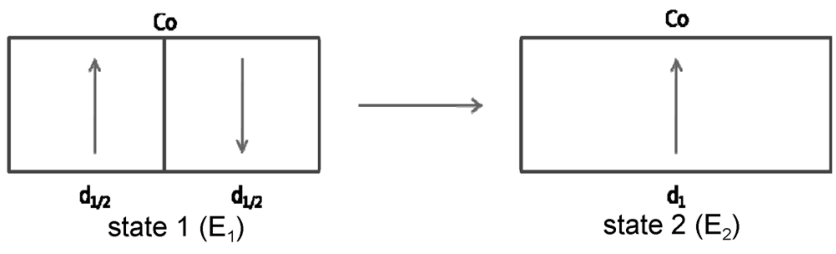

Fig. 6. Two types of wall formations below and above $T_{c}$ of $\mathrm{Co}_{2}$ TiSn Heusler alloy phase in two different energy states: state 1 and state 2 .

The change of coercivity with temperature was modeled using the formation of a wall at the phase boundary in the low temperature region. In order to obtain the relationship between the coercivity change with temperature and the wall energy at the phase boundary, the total magnetic free energy of the exchange coupled equilibrium system is calculated both below and above the $T_{c}$ of the Heusler alloy phase. Below $T_{c}$, the demagnetized state can be defined as state 0 [Fig. 6(a)] and the total free energy is described by

$$
\mathrm{E}_{0}=\gamma_{\mathrm{Heul}}+2 \gamma_{\mathrm{AF}}
$$

where $\gamma_{\text {Heul }}$ is the Bloch wall energy of the Heusler alloy phase and $\gamma_{\mathrm{AF}}$ is the antiferromagnetic wall energy per unit area due to the exchange coupling at the phase boundary. State 1 [Fig. 6(a)] describes that a soft $\mathrm{Co}_{2} \mathrm{TiSn}$ Heusler alloy phase is saturated at a low magnetic field and two antiferromagnetic walls are formed by the negative exchange coupling at the phase boundary. The total free energy is given by

$$
\mathrm{E}_{1}=-\mathrm{M}_{\mathrm{s} 1} \mathrm{~d}_{1} \mathrm{H}+\mathrm{M}_{\mathrm{s} 2} \mathrm{~d}_{2} \mathrm{H}+2 \gamma_{\mathrm{Af}}
$$

where $M_{s i}$ is the saturation magnetization of $i$ phase, $d_{i}$ is the width of $\mathrm{i}$ phase, and $\mathrm{H}$ is the field required to reverse the magnetization. State 2 [Fig. 6(a)] shows the ferromagnetic wall formation at the phase boundary under the saturating field. The ferromagnetic wall $\gamma_{\mathrm{F}}$ can be associated with a saturation negative field. The total free energy of state 2 below $\mathrm{T}_{\mathrm{c}}$ of the Heusler alloy phase can be described by

$$
\mathrm{E}_{2}=\mathrm{M}_{\mathrm{s} 1} \mathrm{~d}_{1} \mathrm{H}+\mathrm{M}_{\mathrm{s} 2} \mathrm{~d}_{2} \mathrm{H}+2 \gamma_{\mathrm{F}} .
$$

The total free energy difference induced by the wall formation at the phase boundary is associated with $\mathrm{H}_{\mathrm{c}, 12}$ [Fig. 6(a)]. From Eq. 2 and Eq. 3, the total free energy difference between state 1 and 2 is described by

$$
\mathrm{E}_{1}-\mathrm{E}_{2}=\left(\gamma_{\mathrm{AF}}-\gamma_{\mathrm{F}}\right) / \mathrm{M}_{\mathrm{s} 1} \mathrm{~d}_{1} \text {. }
$$

The saturation negative field $\left(\mathrm{H}_{\mathrm{c}, 12}\right)$ can be defined as a theoretical coercivity below $\mathrm{T}_{\mathrm{c}}$. Eq. 4 gives the expression:

$$
\mathrm{H}_{\mathrm{c}}^{\text {low }}=\mathrm{H}_{\mathrm{c}, 12}=\left(\gamma_{\mathrm{Af}}-\gamma_{\mathrm{F}}\right) / \mathrm{M}_{\mathrm{s} 1} \mathrm{~d}_{1} \text {. }
$$

Above $\mathrm{T}_{\mathrm{c}}$ of the Heusler alloy phase, the coercivity is determined by the hexagonal Co phase. The total free energies of states 1 and 2 in Fig. 6(b) are given by

$$
\begin{aligned}
& E_{1}=M_{s 1} d_{1 / 2} H-M_{s 1} d_{1 / 2} H+\gamma_{C o} \\
& E_{2}=M_{s 1} d_{1} H
\end{aligned}
$$

where $d_{1 / 2}$ is half the size of the $i$ phase. The total free energy difference above $T_{c}$ of the Heusler alloy phase is

$$
E_{1}-E_{2}=\gamma_{\text {Co }}-M_{s 1} d_{1} H=0 .
$$

Theoretical coercivity is expressed by

$$
\mathrm{H}_{\mathrm{c}}^{\text {high }}=\gamma_{\mathrm{Co}} / \mathrm{M}_{\mathrm{s} 1} \mathrm{~d}_{1} \text {. }
$$

From Eqs. 5 and 9, the change in coercivity with temperature can be described in terms of energy at the phase boundary,

$\left.\mathrm{H}_{\mathrm{c}}=\mathrm{H}_{\mathrm{c}}^{\text {high }}-\mathrm{H}_{\mathrm{c}}^{\text {low }}=\left(\gamma_{\mathrm{Co}} / \mathrm{M}_{\mathrm{s} 1} \mathrm{~d}_{1}\right)-\left[\left(\gamma_{\mathrm{AF}}-\gamma_{\mathrm{F}}\right) / \mathrm{M}_{\mathrm{s} 1} \mathrm{~d}_{1}\right)\right]$.

It can be assumed that the antiferromagnetic wall energy due to the negative exchange coupling is almost zero under a magnetic field. Eq. 10 is simply described by

$$
\mathrm{H}_{\mathrm{c}}=\left(\gamma_{\mathrm{Co}}-\gamma_{\mathrm{F}}\right) / \mathrm{M}_{\mathrm{s} 1} \mathrm{~d}_{1} \text {. }
$$

The change in coercivity $\mathrm{H}_{c}$ can be determined experimentally by extrapolating from the high-temperature region to the low-temperature region in the coercivity curves (Fig. 4). The three curves of $\mathrm{Co}_{3} \mathrm{TiSn}, \mathrm{Co}_{4} \mathrm{TiSn}$, and $\mathrm{Co}_{5} \mathrm{TiSn}$ show almost the same values of $\mathrm{H}_{c}$, which are

Table 1. The values used for the extraction of $\gamma_{F}$ from Eq. 11: $\mathrm{M}_{\mathrm{s} 1}$ : saturation magnetization of hexagonal Co $\left(\mathrm{emu} / \mathrm{cm}^{3}\right) ; \mathrm{M}_{\mathrm{s} 2}$ saturation magnetization of $\mathrm{Co}_{2} \mathrm{TiSn}$ Heusler alloy $\left(\mathrm{emu} / \mathrm{cm}^{3}\right)$. $\mathrm{d}_{1}$ : size of Co matrix, which is the distance between precipitates $(\mathrm{cm}) . \mathrm{d}_{2}$ : size of $\mathrm{Co}_{2} \mathrm{TiSn}$ Heusler alloy precipitate $(\mathrm{cm})$. $\gamma_{\mathrm{Co}_{0}}$ : domain wall energy of hexagonal Co $\left(\mathrm{erg} / \mathrm{cm}^{2}\right)$.

\begin{tabular}{cccccc}
\hline \hline $\mathrm{M}_{\mathrm{s} 1}$ & $\mathrm{M}_{\mathrm{s} 2}$ & $\mathrm{~d}_{1}$ & $\mathrm{~d}_{2}$ & $\gamma_{\mathrm{Co}}$ & $\Delta \mathrm{H}_{\mathrm{c}}$ \\
\hline 1422 & 300 & $2.1 \times 10^{-4}$ & $1.56 \times 10^{-3}$ & 11.3 & 82 \\
\hline
\end{tabular}


$\sim 82 \mathrm{Oe}$. Therefore, the ferromagnetic wall energy $\left(\gamma_{\mathrm{F}}\right)$ can be extracted from Eq. 11. The size of the hexagonal Co matrix, which is the distance between the $\mathrm{Co}_{2} \mathrm{TiSn}$ Heusler alloy precipitates, is approximately obtained from the microstructure of the $\mathrm{Co}_{4} \mathrm{TiSn}$ sample. The values used for the extraction of $\gamma_{\mathrm{F}}$ from Eq. 11 are listed in Table 1. The extracted wall energy at the phase boundary is $\sim 13.2$ $\mathrm{erg} / \mathrm{cm}^{2}$.

\subsection{Giant Magnetoresistance (GMR) effect of $\mathrm{Co} /$ $\mathrm{Co}_{2}$ TiSn Thin Films with Two Phase Magnet}

The x-ray diffraction pattern and SEM picture obtained from an as-deposited $\mathrm{Co} / \mathrm{Co}_{2} \mathrm{TiSn}$ thin film with two magnetic phases that have the composition of $\mathrm{Co}_{5} \mathrm{TiSn}$, shows the formation of stable $\mathrm{Co}_{2}$ TiSn Heusler alloy precipitates in a hexagonal Co matrix. The (100) peak of the hexagonal Co matrix overlaps with the (220) peak of the $\mathrm{Co}_{2} \mathrm{TiSn}$ Heusler alloy precipitate, similarly to the case of the bulk $\mathrm{Co}_{4} \mathrm{TiSn}$ sample with two phases. The primary peaks from the hexagonal Co matrix and $\mathrm{Co}_{2} \mathrm{TiSn}$ Heusler alloy do not shift from their normal position. From the plane view SEM pictures of as-deposited and annealed films, the microstructures of both before and after annealing do not significantly differ and the $\mathrm{Co}_{2} \mathrm{TiSn}$ Heusler alloy precipitates, which have an average size of $7000 \AA$, have a spherical shape in the hexagonal Co matrix. By comparing the average size with the film thickness of approximately $5000 \AA$, it is conceivable that the precipitates are elongated normal to the film plane and that the $\mathrm{Co}_{2} \mathrm{TiSn}$ Heusler alloy precipitates form a texture structure with the Co matrix. Figs. 7(a) and 7(b) show the in-plane and out-of-plane magnetization hysteresis loops, respectively, of the as-deposited $\mathrm{Co} / \mathrm{Co}_{2} \mathrm{TiSn}$ thin film. The magnetization curve with the applied field perpendicular to the film plane demonstrates a typical bubble domain hysteresis loop, which implies the perpendicular easy axis for magnetization. It is expected that this perpendicular easy axis will be induced by the columnar structure in the film and by the antiparallel exchange coupling between the $\mathrm{Co}_{2} \mathrm{TiSn}$ precipitates and the $\mathrm{Co}$ matrix, where they have a direction normal to the film plane [6]. Although there is no microstructural evidence for the formation of a columnar structure in the Co matrix, the formation of a columnar structure in sputtered films is frequently observed. Both in-plane and out-ofplane magnetization curves show marginal opening with some coercivity in a low field, which indicates that the magnetization is caused by two magnetic phases. By considering the volume and magnetization fraction of each phase in the $\mathrm{Co}_{5} \mathrm{TiSn}$ composition that has two phases, it can be seen that the $25 \%$ magnetization is
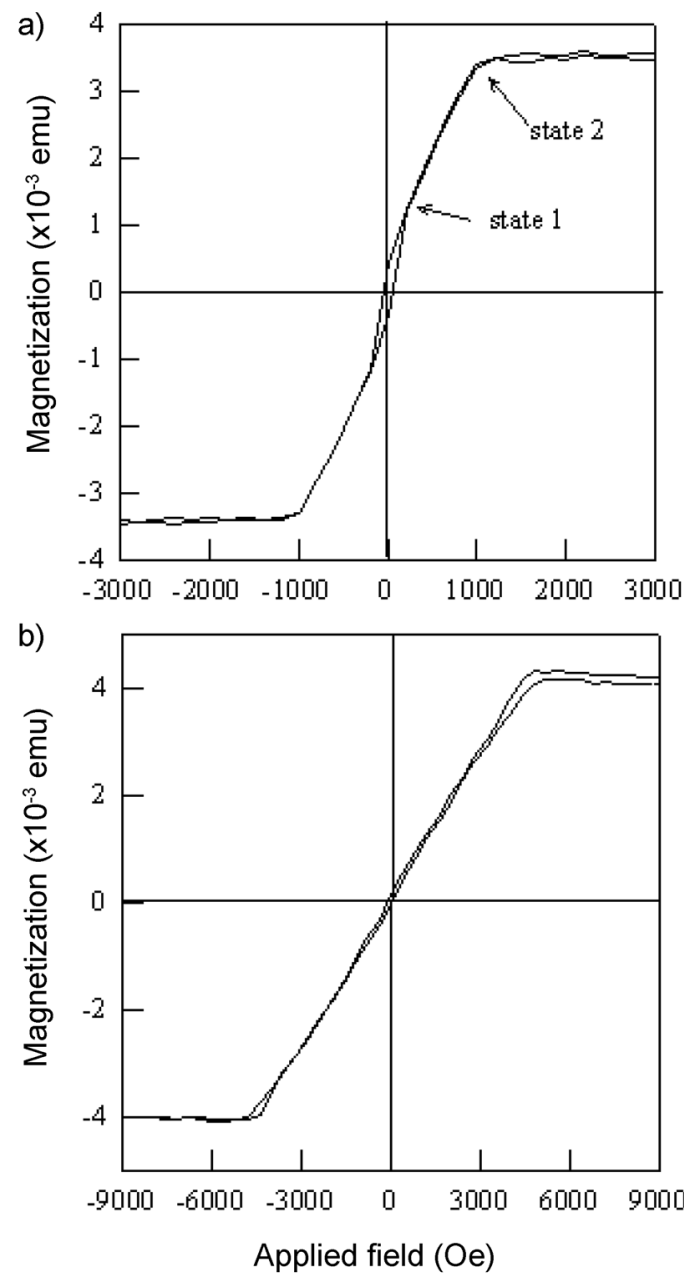

Fig. 7. Magnetization hysteresis loops of as-deposited sample: a) in-plane magnetization curve, b) out-of-plane magnetization curve.

capable of being generated by the $\mathrm{Co}_{2} \mathrm{TiSn}$ Heusler alloy precipitates. This value is close to the $23 \%$ magnetization fraction of the open loop portion obtained from Fig. 7a, which indicates that the magnetization at a low field is carried out by the $\mathrm{Co}_{2}$ TiSn Heusler alloy precipitates. The magnetization process in the films with a two-phase magnet can be explained through the model proposed in the previous section (Sec. 3.3). The shape of the in-plane magnetization curve of the film with a two-phase magnet differs from those of other bubble domain materials, which are usually S-shaped. When the field is applied, the soft $\mathrm{Co}_{2}$ TiSn Heusler alloy precipitates are magnetized in a low field and all spins line up along the field direction (Fig. 6a, state 1). Subsequently, as the field increases, the spins in the columnar structure of the Co matrix that are perpendicular to the plane are rotated and the antiparallel exchange coupled spins at the phase boundary are also rotated by the field. It then starts to form a ferromagnetic 

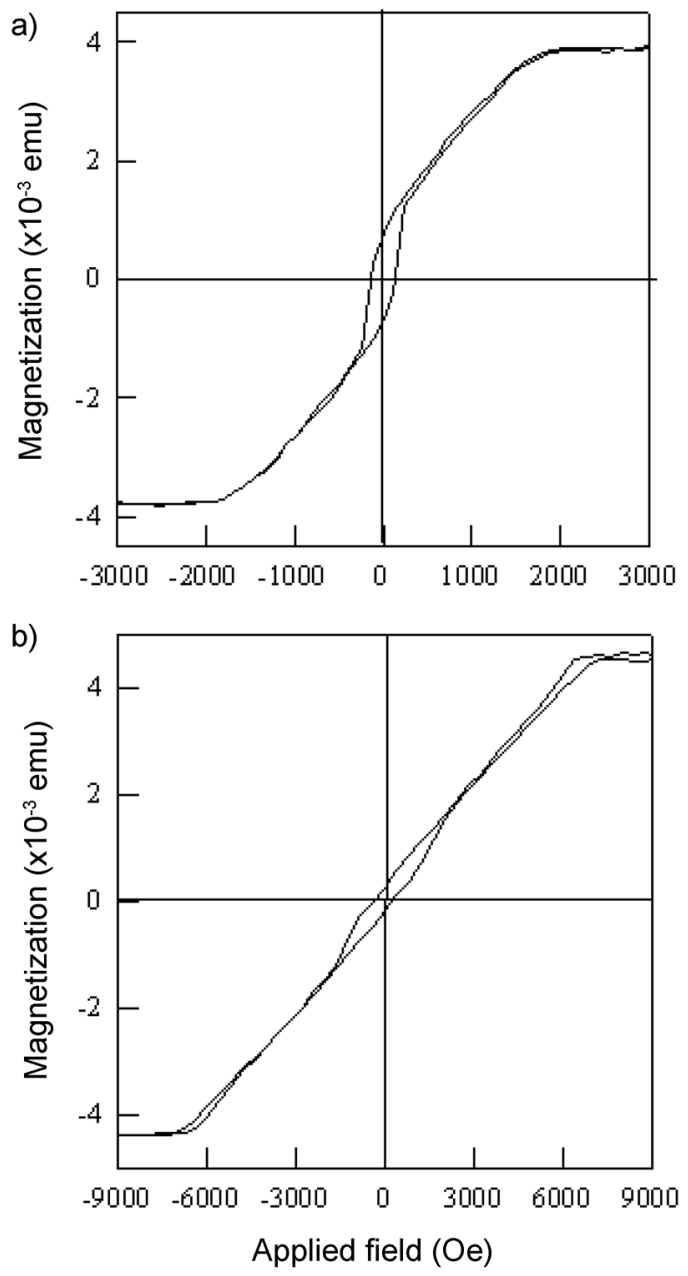

Fig. 8. Magnetization hysteresis loops of the sample annealed in vacuum: a) in-plane magnetization curve, b) out-of-plane magnetization curve.

wall $\left(\gamma_{\mathrm{F}}\right)$ and the magnetization linearly increases with the field. Under a high saturation field $\left(\mathrm{H}_{\text {sat }}\right)$, the sample is fully magnetized and all spins in both phases line up along the field direction with a ferromagnetic wall at the phase boundary (Fig. 6a, state 2). Fig. 8 and Fig. 9 show the in-plane and out-of-plane magnetization curves of the samples that were annealed in a vacuum and an $\mathrm{N}_{2}$ gas, which have almost the same shapes. In annealed samples, the opening at the in-plane and out-of-plane magnetization curves and the coercivity are increased. The saturation fields $\left(\mathrm{H}_{\mathrm{sat}}\right)$ are increased from 1200 Oe to $2000 \mathrm{Oe}$ for the in-plane magnetization curve and from 5000 Oe to 7000 Oe for the out-of-plane magnetization curve. Even though the chemical ordering of $\mathrm{Co}_{2} \mathrm{TiSn}$ Heusler alloy precipitates is not represented with clarity, the increase of $\mathrm{H}_{\text {sat }}$ and the opening of the hysteresis loops in the annealed samples are probably due to the increase in the number of exchange coupling sites at the phase boundary. This is
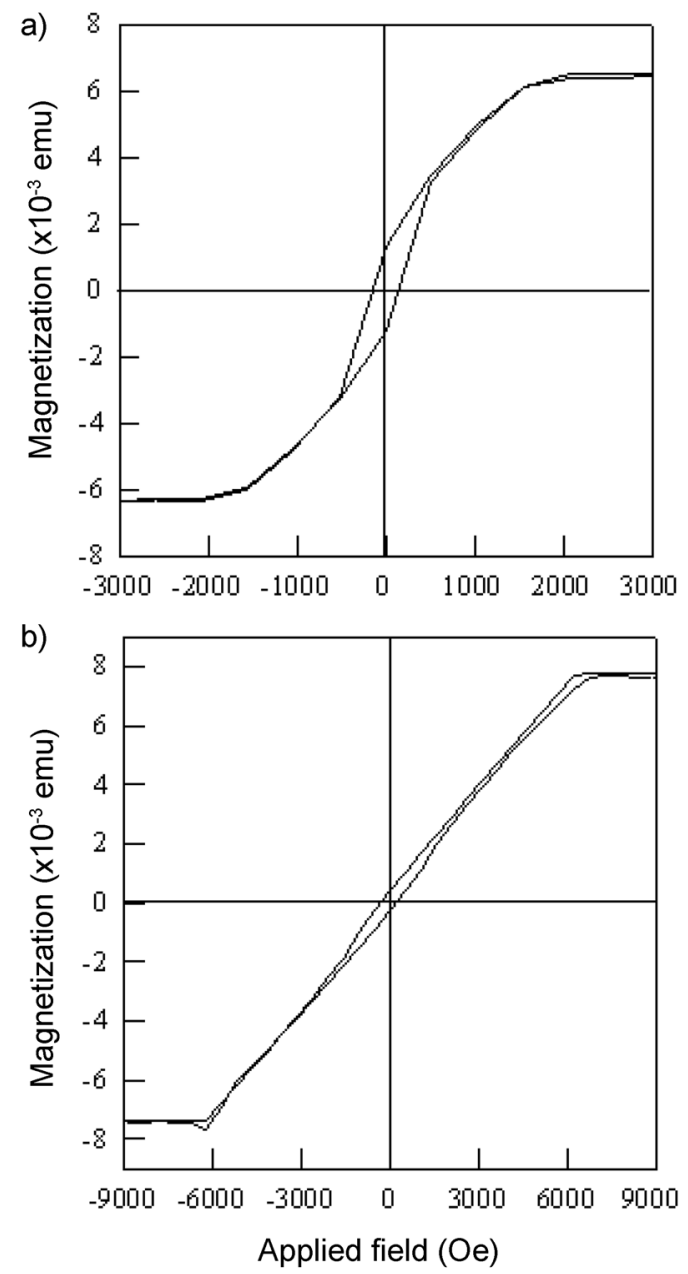

Fig. 9. Magnetization hysteresis loops of the sample annealed in $\mathrm{N}_{2}$ gas: a) in-plane magnetization, b) out-of-plane magnetization.

caused by the increase in the number of possible Co-Co exchange interactions in the Heusler alloy structure induced by the change of chemical ordering from disorder to order [7]. The $\mathrm{Co}_{2}$ TiSn Heusler alloy has eight magnetic Co sites and eight non-magnetic $\mathrm{Ti}$ and $\mathrm{Sn}$ sites in the $\mathrm{L} 21^{-}$type structure, where the magnetic moments are associated with the ordered Co sites. The Curie temperature of the Heusler alloy increases with an increase in the magnetic Co sites in the lattice [7]. Figs. 10(a) and 10(b) exhibit saturation magnetization changes of the film that has a two-phase magnet as a function of temperature before and after annealing in a vacuum. The $\mathrm{T}_{\mathrm{c}}$ of the low Curie temperature $\mathrm{Co}_{2} \mathrm{TiSn}$ precipitate is estimated by extrapolation from the break in the magnetization curves. The break point of the magnetization curve before annealing occurs at approximately $170^{\circ} \mathrm{C}$, while after annealing it occurs at a slightly higher temperature of $200^{\circ} \mathrm{C}$. Both of these temperatures are significantly higher than the 
a)

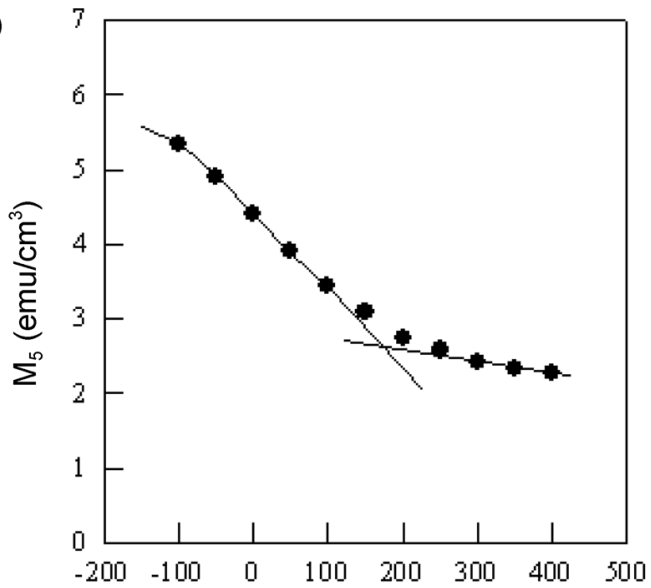

b)

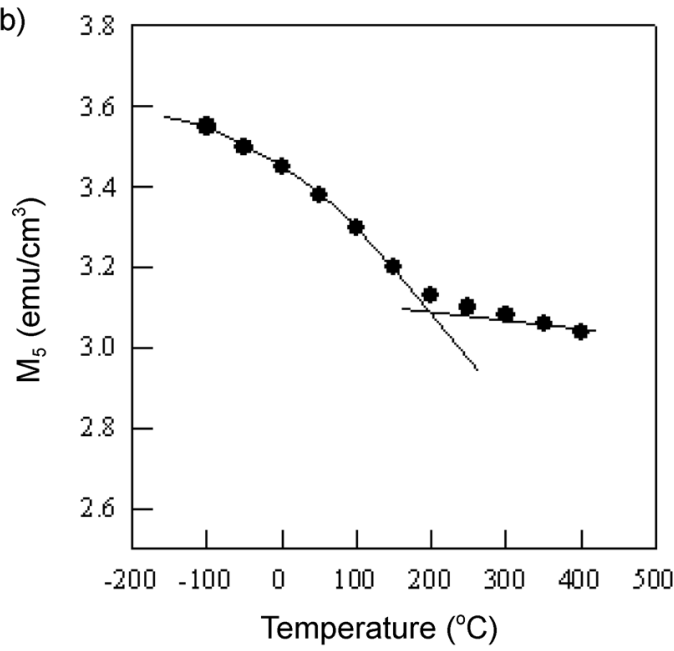

Fig. 10. Changes of saturation magnetization, Ms, as a function of temperature: a) as-deposited, b) annealed in vacuum.

Curie temperature of $\sim 100^{\circ} \mathrm{C}$, of the arc-melted bulk single phase $\mathrm{Co}_{2} \mathrm{TiSn}$. This indicates that extra magnetic Co sites occupy non-magnetic $\mathrm{Ti}$ or $\mathrm{Sn}$ sites in the $\mathrm{Co}_{2} \mathrm{TiSn}$ Heusler alloy precipitates. The saturation magnetization change of the annealed film is less sensitive to temperature in the low temperature region than that of the asdeposited film, while the Curie temperature is slightly higher in the annealed film. These results can be explained by the increase of the number of Co-Co exchange interactions in the Heusler alloy structure resulting from the change of chemical ordering due to annealing. The $\mathrm{Co} / \mathrm{Co}_{2} \mathrm{TiSn}$ thin films with a two-phase magnet also show an unusual temperature dependence of the coercivity (Fig. $11 \mathrm{a}$ and $\mathrm{b}$ ).

The GMR effect of $\mathrm{Co} / \mathrm{Co}_{2} \mathrm{TiSn}$ thin films that have a two-phase magnet with the composition of $\mathrm{Co}_{5} \mathrm{TiSn}$ was observed in the annealed samples. Figs. 12(a) 13(b) illustrate various features of electrical resistivity occurring at room temperature of the $\mathrm{Co} / \mathrm{Co}_{2} \mathrm{TiSn}$ system that was
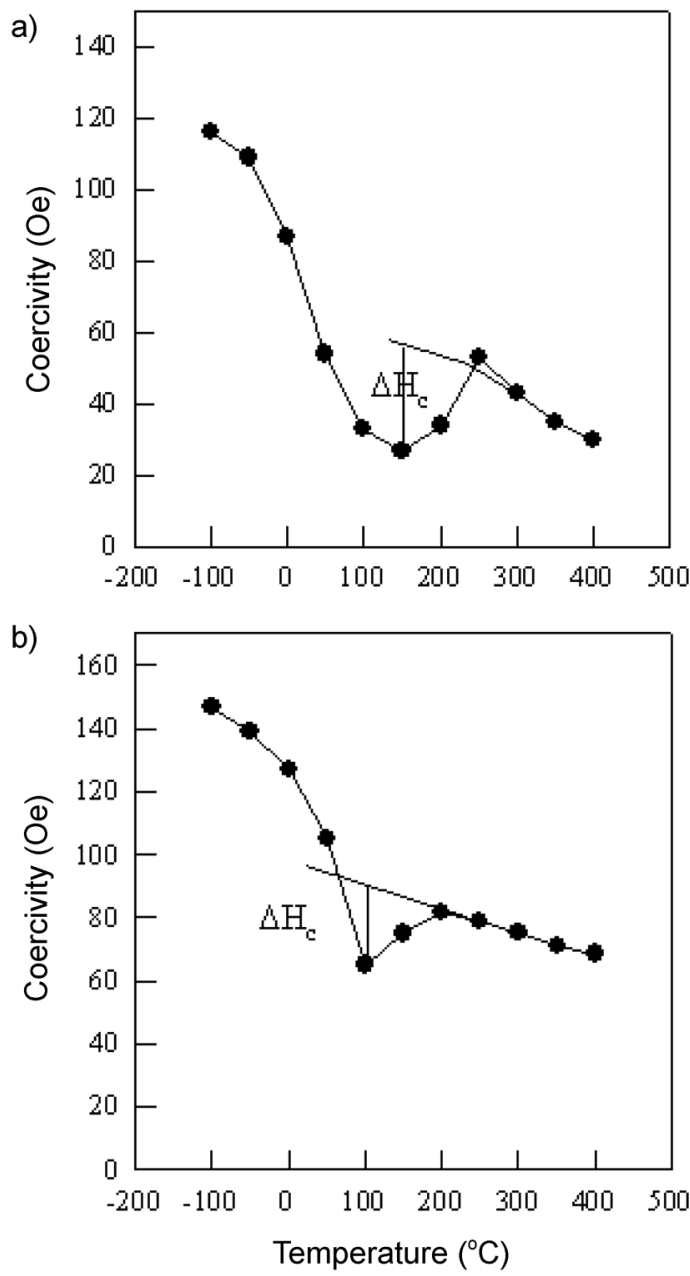

Fig. 11. Change of coercivity as a function of temperature: a) as-deposited, b) annealed in vacuum.

annealed at different conditions. The resistivity at room temperature of the sample that was annealed in a vacuum is $3 \times 10^{-5} \Omega \mathrm{cm}$, which is less than $5 \times 10^{-5} \Omega \mathrm{cm}$ of the as-deposited samples. When the sample is annealed in an $\mathrm{N}_{2}$ gas atmosphere, the resistivity increased to $8 \times 10^{-5}$ $\Omega \mathrm{cm}$, which was significantly higher than the resistivity of the as-deposited sample. It was considered that the resistivity of the Co matrix was as small as $5.6 \times 10^{-6}$ $\Omega \mathrm{cm}$ and that the Co had no solid solubility for Ti and Sn at room temperature. It was also assumed that there was not a significant difference in resistivity between the disordered and ordered structures of the $\mathrm{Co}_{2} \mathrm{TiSn}$ Heusler alloy precipitates. Consequently, it can be inferred that the reason for the higher resistivity of the as-deposited film over that of the film annealed in a vacuum is probably due to the segregation of highly resistive $\mathrm{Ti}\left(3.9 \times 10^{-5}\right.$ $\Omega \mathrm{cm})$ and $\mathrm{Sn}\left(1.15 \times 10^{-5} \Omega \mathrm{cm}\right)$ along the columnar and phase boundaries. In the sample that was annealed in $\mathrm{N}_{2}$ gas, there is no apparent evidence for a much higher 

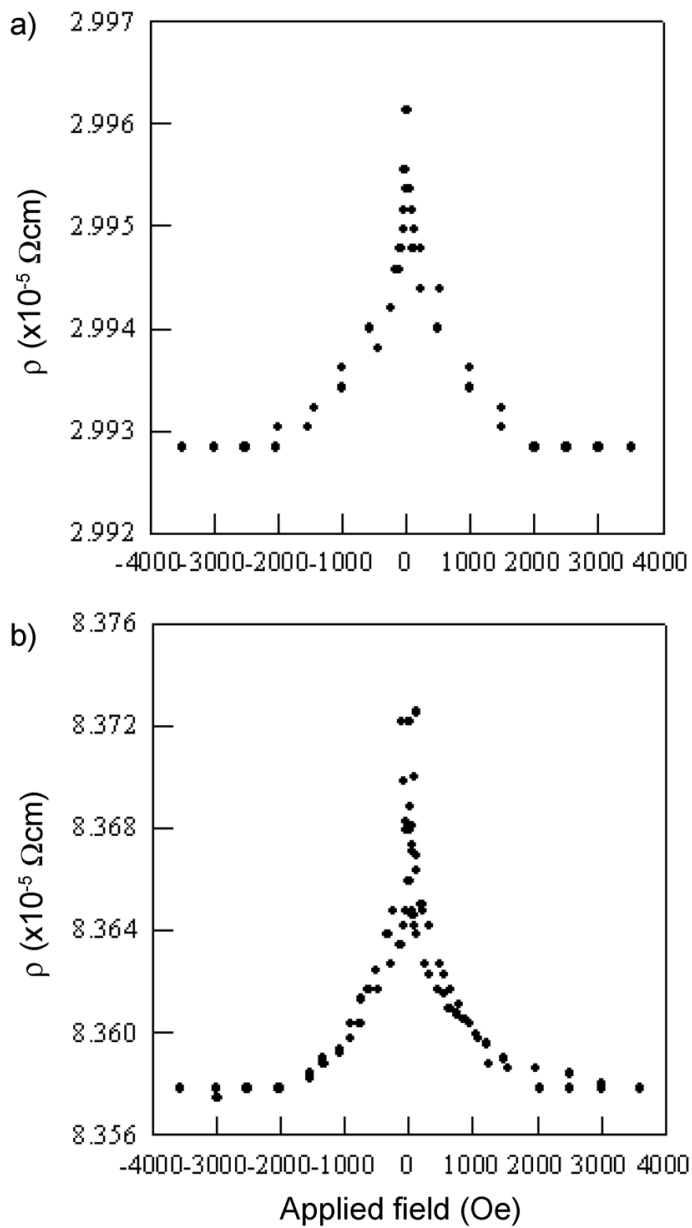

Fig. 12. Resistivity changes vs. applied field at room temperature in $\mathrm{Co} / \mathrm{Co}_{2} \mathrm{TiSn}$ thin films: a) annealed in vacuum, b) annealed in $\mathrm{N}_{2}$ gas.

resistivity. However, it can be also inferred that the high resistive $\mathrm{Ti}$ and $\mathrm{Sn}$ atoms segregated at the columnar boundary form a higher resistive nitride (TiN: $2 \times 10^{-4}$ $\Omega \mathrm{cm}$ ) with a diffused nitrogen atom. Furthermore, a part of Ti and Sn atoms segregated at the phase boundary are also dissolved into the Heusler alloy precipitate and form a chemical ordering in the Heusler alloy lattice. The annealing effects on resistivity could be related to the magnetization change with temperature before and after annealing. Fig. 12(a) shows the resistivity change at room temperature, as a function of the applied field in the film that was annealed in a vacuum, while Fig. 12(b) shows that for the film annealed in $\mathrm{N}_{2}$ gas. For both curves, the negative resistance change occurred when the field increased and was saturated at a field of around $2 \mathrm{kOe}$, which is the same result as that for the in-plane saturation magnetization field. The magnetoresistivity changes of $\mathrm{dr}(\mathrm{H})$ with the field are shown in Figs. 13(a) and 13(b). In this study, the giant magnetoresistance shown in Fig.
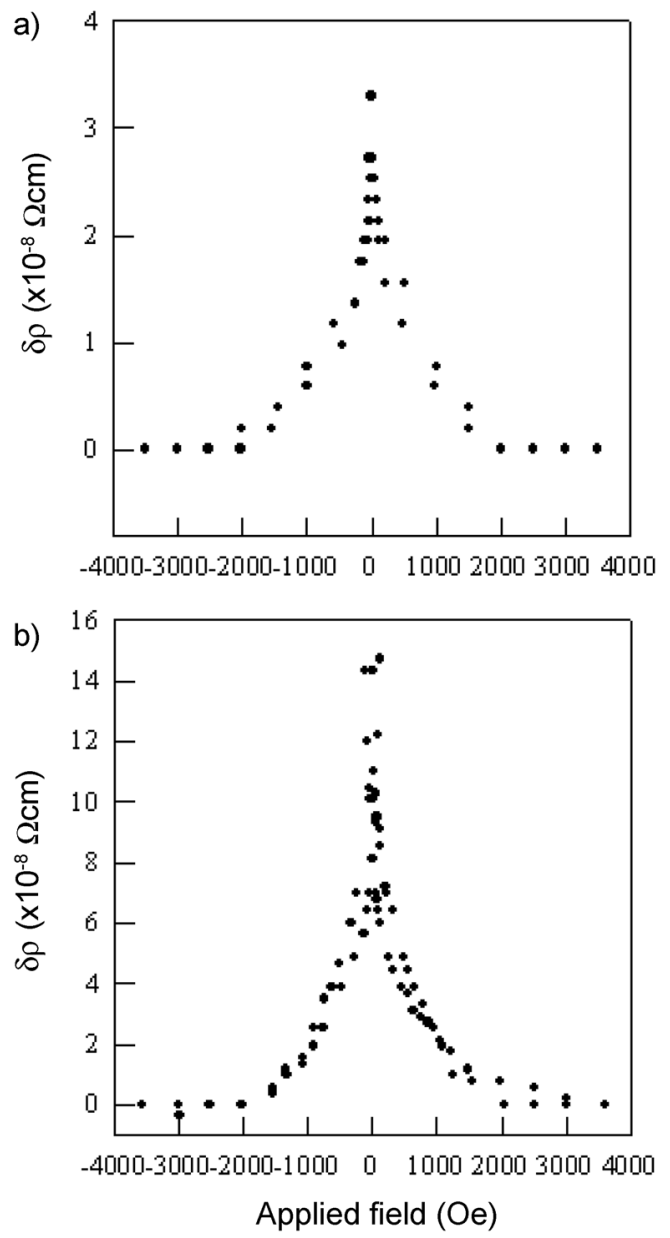

Fig. 13. Magnetoresistivity changes vs. applied field at room temperature in $\mathrm{Co} / \mathrm{Co}_{2} \mathrm{TiSn}$ thin films: a) annealed in vacuum, b) annealed in $\mathrm{N}_{2}$ gas.

12(a) and 12(b) are defined as

$$
\mathrm{GMR}=\delta \rho / \rho=\left[\rho(\mathrm{H})-\rho\left(\mathrm{H}_{\max }\right)\right] / \rho\left(\mathrm{H}_{\max }\right)
$$

where $\rho\left(\mathrm{H}_{\text {max }}\right)$ is the resistivity at the saturation field. In the film annealed in $\mathrm{N}_{2}$ gas, the $\delta \rho(\mathrm{H})$ is approximately $1.43 \times 10^{-7} \Omega \mathrm{cm}$ and the GMR is $0.18 \%$. In the film annealed in a vacuum, the $\delta \rho(\mathrm{H})$ and GMR are approximately $3.3 \times 10^{-8} \Omega \mathrm{cm}$ and $0.12 \%$, respectively. Even though the film annealed in $\mathrm{N}_{2}$ gas has a higher resistivity, the GMR is larger than that of the film annealed in a vacuum. In the $\mathrm{Co} / \mathrm{Co}_{2} \mathrm{TiSn}$ thin films with a two-phase magnet, the field dependence of magnetoresistance is the same whether the field is applied perpendicular or parallel to the current, similarly to the GMR effect in other GMR systems. This is not true of the anisotropy magnetoresistance (AMR) effect. In the $\mathrm{Co} / \mathrm{Co}_{2} \mathrm{TiSn}$ system, the field dependence is the same even if the field is applied perpendicular to the film plane, with the exception, as expected, for demagnetization effects. Fig. 14 shows the 


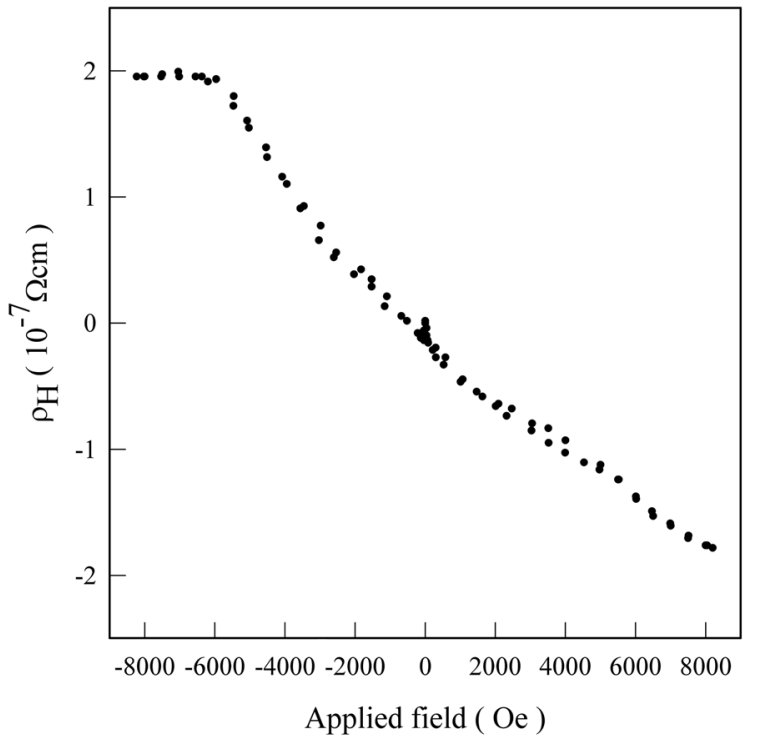

Fig. 14. Negative spontaneous Hall resistivity of $\mathrm{Co} / \mathrm{Co}_{2} \mathrm{TiSn}$ thin film with two-phase magnet annealed in $\mathrm{N}_{2}$ gas.

negative spontaneous Hall resistivity of the $\mathrm{Co} / \mathrm{Co}_{2} \mathrm{TiSn}$ film that was annealed in $\mathrm{N}_{2}$ gas. The Hall hysteresis loop is unbalanced at the positive and negative field regions and has a different shape from that of the magnetization hysteresis loop. The saturation of Hall resistivity in the negative field direction is due to the decrease of Hall Voltage that is affected by the negative magnetoresistance. The magnetoresistance effect, which arises from the domain walls in ferromagnets due to the spontaneous Hall effect, is described by [8]

$$
\begin{aligned}
& \delta \rho / \rho_{\mathrm{s}}=\mathrm{C}|\beta|^{2} \\
& \beta=\tan \theta_{\mathrm{H}}=\rho_{\mathrm{H}} / \rho
\end{aligned}
$$

where $\mathrm{C}$ is a constant of order unity and $\beta$ is the tangent of the Hall angle. The spontaneous Hall effect in the $\mathrm{Co} /$ $\mathrm{Co}_{2}$ TiSn system is relatively small. At $7 \mathrm{kOe}, \rho_{\mathrm{H}}$ is $2 \times$ $10^{-7} \Omega \mathrm{cm}$ and $\beta^{2}$ is $6.25 \times 10^{-6}$. It is therefore expected that the magnetoresistance effect arising from the domain walls is infinitesimally small in these materials. Therefore, the negative GMR of the $\mathrm{Co} / \mathrm{Co}_{2} \mathrm{TiSn}$ thin films with a two-phase magnet could be related to the spin scattering caused by the antiferromagnetic exchange coupling at the phase boundary. The magnetic scattering process can be modeled with relation to the model based on the unusual change in coercivity according to temperature (Fig. 6). Fig. 15 shows a simple schematic diagram of the magnetic scattering process of the conduction electron in the $\mathrm{Co} /$ $\mathrm{Co}_{2}$ TiSn system. The $\mathrm{Co} / \mathrm{Co}_{2} \mathrm{TiSn}$ thin films with a twophase magnet consist of a soft ferromagnetic $\mathrm{Co}_{2} \mathrm{TiSn}$ Heusler alloy precipitate orientated antiparallel to the Co matrix due to the negative exchange at the $\mathrm{Co} / \mathrm{Co}_{2} \mathrm{TiSn}$ a)

$\mathrm{H}=\mathbf{0}$
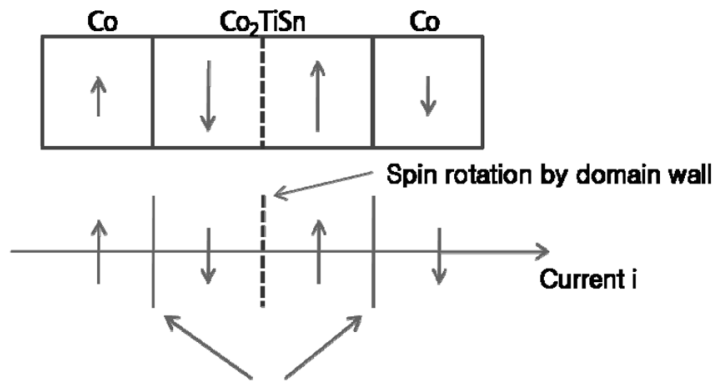

Large magnetic scattering by antiferro Exchange coupling

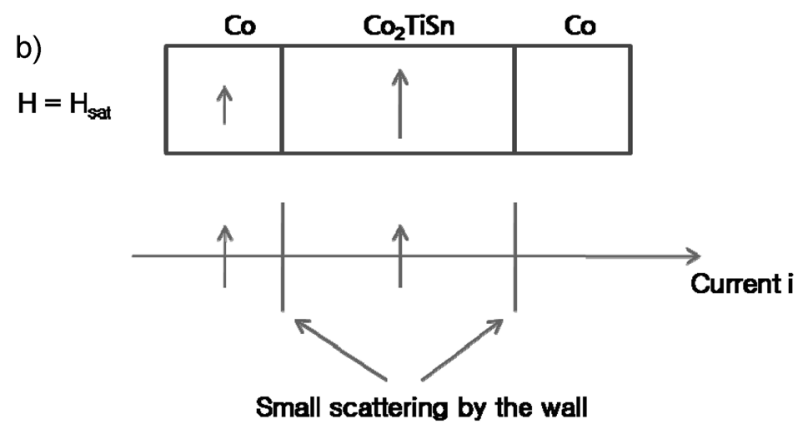

Fig. 15. Schematic diagram of the magnetic scattering process of conduction electron in $\mathrm{Co} / \mathrm{Co}_{2} \mathrm{TiSn}$ system $(\mathrm{a} ; \mathrm{H}=0, \mathrm{~b}$; $\left.\mathrm{H}=\mathrm{H}_{\mathrm{sat}}\right)$.

phase boundary. When the field is not applied, the polarized conduction electrons from the ferromagnetic Co matrix are antiparallel to the $\mathrm{Co}_{2} \mathrm{TiSn}$ Heusler alloy precipitates. They are also scattered by the $\mathrm{Co}_{2} \mathrm{TiSn}$ spins that are directed in reverse order at the phase boundary as the polarized electrons traverse the $\mathrm{Co} / \mathrm{Co}_{2} \mathrm{TiSn}$ interface. After the scattering process, the spin orientation of the electrons lines up with the $\mathrm{Co}_{2} \mathrm{TiSn}$ spin orientation. The electrons are then smoothly and gradually polarized by the domain wall of the $\mathrm{Co}_{2} \mathrm{TiSn}$ Heusler alloy without large scattering. When the electrons traverse the $\mathrm{Co} / \mathrm{Co}_{2} \mathrm{TiSn}$ interface from the $\mathrm{Co}_{2} \mathrm{TiSn}$ precipitate to the Co matrix, the scattering proceeds in the same way. Therefore, the scattering event occurs twice as the conduction electrons traverse through the $\mathrm{Co}_{2} \mathrm{TiSn}$ precipitates, which cause the high resistivity without an applied field. As the field increases, the $\mathrm{Co}_{2} \mathrm{TiSn}$ precipitate turns into the saturation field $\left(\mathrm{H}_{\text {sat }}\right)$. Consequently, the polarized conduction electrons from the Co matrix pass through the $\mathrm{Co}_{2} \mathrm{TiSn}$ precipitate without any large scattering so that the resistivity decreases. After saturation, the ferromagnetic wall $\left(\gamma_{\mathrm{F}}\right)$ formed at the phase boundary yields a positive $\delta \rho$. However, from the experimental data and the model, it is considered that the GMR effect in the $\mathrm{Co} / \mathrm{Co}_{2} \mathrm{TiSn}$ thin films that have a twophase magnet is dominated by a negative $\delta \rho$ and is attributed to the spin scattering by the antiparallel exchange 
coupling at the $\mathrm{Co} / \mathrm{Co}_{2} \mathrm{TiSn}$ phase boundary.

\section{Conclusion}

A composite magnet, $\mathrm{Co} / \mathrm{Co}_{2} \mathrm{TiSn}$, consisting of $\mathrm{Co}_{2} \mathrm{TiSn}$ Heusler alloy precipitates in a hexagonal Co matrix, and is formed in the 60 78 atomic \% of a Co composition. This system shows an unusual coercivity change with a concurrent change in temperature. The relationship between the change in coercivity with temperature and the wall energy at the phase boundary is described by a model, where it is assumed that the formation of wall is at the phase boundary, thus making it possible to extract the value of $\gamma_{\mathrm{F}}$.

The annealed $\mathrm{Co} / \mathrm{Co}_{2} \mathrm{TiSn}$ thin films with a two-phase magnet display $0.12 \%$ GMR effect, which indicates the scattering of polarized conduction electrons due to the antiparallel exchange coupling at the phase boundary. This system also has an unusual coercivity change with temperature and the magnetization process can be explained by the model proposed in the bulk $\mathrm{Co} / \mathrm{Co}_{2} \mathrm{TiSn}$ two-phase magnet. Both the as-deposited and the annealed films show higher Curie temperatures, which may be caused by the increase of magnetic Co sites in the $\mathrm{Co}_{2} \mathrm{TiSn}$ Heusler alloy precipitates. The Curie temperature of the annealed film was slightly higher than that of the asdeposited film, which may indicate that the precipitates in the annealed film are highly ordered. The scattering process of conduction electrons at the phase boundary is modeled on the basis of the magnetization process.

\section{Acknowledgements}

This work was supported by the National Program for Tera-level Nano devices as a 21st Century Frontier Program.

\section{References}

[1] E. F. Kneller and R. Hawig, IEEE trans. Magn., 27, 3588 (1991).

[2] Chun-Yeol You, J. of the Korean Magnetics Society, 15(4), 221 (2005).

[3] T. W. Kim and R. J. Gambino, J. Appl. Phys., 79(8), 5253 (1996).

[4] T. W. Kim, R. J. Gambino, and T. R. McGuire, J. Appl. Phys., 89(11), 7299 (2001).

[5] Taewan Kim and Sung-Chul Shin, Proceedings of the Korean Magnetics Society Conference, y1998m5a, 116 (1998).

[6] J. Chang, B. A. Gribkov, H. Kim, H. Koo, S. H. Han, V. L. Mironov, and A. A. Fraerman, J. Magnetics, 12(1), 17 (2007).

[7] P. J. Webster, J. Phys. Chem. Solids, 32, 1221 (1971).

[8] L. Berger, J. Appl. Phys., 49, 2156 (1978). 